Regel der Bundeseinfluss auf das individuelle Wahlverhalten überdurchschnittlich groß. Zwei Effekte moderieren diesen Einfluss. Für Parteien der Landesregierung ist die Wirkung des Bundestagswahlkampfs deutlich abgeschwächt bis vollständig nivelliert. Landesverbänden kleiner Parteien gelingt es offenbar schlechter, sich von den jeweiligen Bundesparteien abzuheben, wenn Bundestagswahlen stattfinden. Zwar bestätigen sich teilweise die bisherigen Befunde zur Einzigartigkeit jeder Landtagswahl, aber mit dem Bundestagswahlkampf wurde eine neue Variable mit systematischem Einfluss auf die individuelle Landtagswahlentscheidung gefunden, und für die Regierungskonstellation und die Größe der Parteien konnten bisherige Befunde präzisiert werden.

Auch wenn die Ergebnisse keine Aussage darüber zulassen, wer genau von zusammenfallenden Wahlen bisher profitierte, so ist doch die grundlegende Frage angebracht, ob ein verstärkter Bundeseinfluss oder eher eine weitgehende Eigenständigkeit von Landtagswahlen politisch gewollt ist. Tabelle 1 legt nahe, dass das mittlerweile regelmäßig auftretende Szenario paralleler Wahlen vor 1990 nur als Ausnahmeereignis bedingt durch vorgezogene Neuwahlen vorkam. Durch die Verlängerung der Wahlperioden in den meisten Bundesländern auf fünf Jahre könnte es in Zukunft immer häufiger dazu kommen, dass Landtagswahlen systematisch im Bundestagswahlkampf stattfinden. Dadurch würde ihre Eigenständigkeit zumindest graduell beeinträchtigt. Insofern sollten die gefundenen Muster bei zukünftigen Koordinierungsversuchen von Wahlterminen berücksichtigt werden.

\title{
Die Bedeutung bestimmter Ministerien, Geschäftsbereiche und Politikfelder für die Parteien in den deutschen Bundesländern
}

\author{
Eric Linhart und Jana Windwehr ${ }^{1}$
}

Zu den am besten empirisch abgesicherten Erkenntnissen der quantitativen Koalitionsforschung gehört William A. Gamsons ${ }^{2}$ Hypothese, dass Parteien die in Koalitionsregierungen zu verteilenden Ämter proportional zu ihren Sitzstärken im Parlament aufteilen. Da dies auch die höchst plausible Vermutung in der politischen Alltagswelt ist, bestätigt sich seine Hypothese in zahlreichen Untersuchungen - wenig überraschend - sowohl im internationalen Vergleich ${ }^{3}$ als auch speziell für deutsche Koalitionsregierungen auf Bundes- und Lan-

1 Dieser Beitrag entstand im Rahmen des von der DFG geförderten Projekts „Ämter- und PolicyMotivation von Parteien bei der Bildung von Koalitionsregierungen “. Die Autoren danken der DFG für die finanzielle Förderung. Dank gilt ebenfalls Johannes Raabe für seine Unterstützung bei der Datenaufbereitung sowie der Redaktion der Zeitschrift für Parlamentsfragen für wertvolle Verbesserungsvorschläge.

2 Vgl. William A. Gamson, A Theory of Coalition Formation, in: American Sociological Review, 26. Jg. (1961), H. 3, S. $373-382$.

3 Vgl. Eric C. Browne / John P. Frendreis, Allocating Coalition Payoffs by Conventional Norm: An Assessment of the Evidence from Cabinet Coalition Situations, in: American Journal of Political Science, 24. Jg. (1980), H. 4, S. 753 - 768; Paul V. Warwick / James N. Druckman, Portfolio Salience and the Proportionality of Payoffs in Coalition Governments, in: British Journal of Political 
desebene. ${ }^{4}$ Trotz dieser Ergebnisse sprechen auch belastbare Erkenntnisse dafür, dass eine solche rein quantitative Sichtweise zu kurz greift. So lässt sich begründet vermuten, dass Schlüsselministerien wie das Finanz-, das Außen- oder das Innenministerium allgemein als bedeutender angesehen werden als andere Portfolios. ${ }^{5}$ Daneben kann sich die Bedeutung von Ressorts im Laufe der Zeit ändern und davon abhängen, welche politischen Themen auf der Agenda stehen.

Neben dem Zeitfaktor variiert die Bedeutung einzelner Portfolios auch über politische Systeme und deren Ebenen hinweg. Dies ergibt sich schon rein logisch aus unterschiedlichen Zuständigkeiten. So kann für die Bundesrepublik etwa angenommen werden, dass die Bereiche Kultus und Bildung auf Landesebene eine vergleichsweise höhere Bedeutung besitzen als auf Bundesebene, da Schul- und Bildungspolitik Angelegenheiten der Länder sind. Außerdem ist zu vermuten, dass bei der Bewertung der Bedeutung von Portfolios auch Unterschiede zwischen Parteien bestehen. Dass die SPD als Arbeiterpartei das Arbeitsministerium als überdurchschnittlich bedeutend ansehen könnte, während die Grünen das Umweltressort als besonders relevant erachten, liegt nahe. Ob diese Vermutungen alle zutreffen, ist damit zunächst nicht gesagt.

\section{Die wenig beachtete Fragestellung parteispezifischer Interessen bei der Verteilung von Ämtern - zum Stand der Literatur}

Fest steht, dass eine erhebliche Diskrepanz besteht zwischen der Anzahl an Beiträgen, die sich mit der quantitativen Aufteilung von Ämtern beschäftigen, und denen, die sich stärker mit dem qualitativen Aspekt befassen. Der Frage unterschiedlicher Interessen der Parteien an den verschiedenen Ämtern wurde bisher kaum nachgegangen. So stellen auch Paul Warwick und James Druckman fest, dass sich bei der Frage „which and how many portfolios each party will receive" 6 , die meisten Beiträge mit dem , how many' beschäftigen, die wenigsten mit dem ,which'. Zu nennen sind hier im Wesentlichen fünf: Im Rahmen von Expertenbefragungen lassen Michael Laver und W. Ben Hunt unter anderem auch die Bedeutung unterschiedlicher Portfolios im Ländervergleich einschätzen. ${ }^{7}$ Obwohl seinerzeit innovativ und wegweisend, kann das Vorgehen nicht unkritisch gesehen werden. ${ }^{8}$ So fehlt etwa eine

Science, 31. Jg. (2001), H. 4, S. 672 - 649; dies., The Portfolio Allocation Paradox: An Investigation into the Nature of a very Strong but Puzzling Relationship, in: European Journal of Political Research, 45. Jg. (2006), H. 4, S. 635 - 665; Royce Carroll / Gary W. Cox, The Logic of Gamson's Law: Pre-Electoral Coalitions and Portfolio Allocations, in: American Journal of Political Science, 51. Jg. (2007), H. 2, S. $300-313$.

4 Vgl. Helmut Norpoth, The German Federal Republic: Coalition Government at the Brink of Majority Rule, in: Eric C. Browne / John Dreijmanis (Hrsg.), Government Coalitions in Western Democracies, New York 1982, S. 7 - 32; Eric Linhart / Franz U. Pappi / Ralf Schmitt, Die proportionale Ministerienaufteilung in deutschen Koalitionsregierungen: Akzeptierte Norm oder das Ausnutzen strategischer Vorteile?, in: PVS, 49. Jg. (2008), H. 1, S. $46-67$.

5 Vgl. Paul V. Warwick / James N. Druckman, Portfolio Salience and the Proportionality of Payoffs in Coalition Governments, a.a.O. (Fn. 3).

6 Ebenda, S. $628 \mathrm{f}$.

7 Vgl. Michael Laver / W. Ben Hunt, Policy and Party Competition, New York 1992.

8 Siehe zur Kritik auch Paul V. Warwick / James N. Druckman, Portfolio Salience and the Proportionality of Payoffs in Coalition Governments, a.a.O. (Fn. 3). 
Einschätzung der Bedeutung des Regierungschefs als Vergleichsbasis, und eine Vielzahl von Portfolios, die die Autoren von vornherein als weniger bedeutend einschätzen, wird erst gar nicht in die Befragung einbezogen. Zudem heben Laver / Hunt ausschließlich auf die allgemeine Bedeutung von Portfolios ab und ignorieren mögliche parteispezifische Unterschiede.

Warwick / Druckman selbst führen eine Überprüfung von Gamsons „Gesetz“ durch, bei der sie im Gegensatz zu früheren Arbeiten die unterschiedliche Bedeutung von Ressorts berücksichtigen. ${ }^{9}$ Hierbei referieren sie die Expertenbefragungen von Laver / Hunt und erstellen für jede der zwölf westeuropäischen Demokratien, die sie untersuchen, eine Reihenfolge der Ämter von hoher bis zu niedriger Bedeutung. ${ }^{10} \mathrm{Im}$ Ergebnis erweisen sich die Analysen mit gewichteten und ungewichteten Portfolios als „extremely similar“11, so dass erstmals zu hinterfragen ist, ob es Parteien möglicherweise doch weniger darum geht, welche Ämter sie besetzen als vielmehr um die reine Anzahl.

Im Gegensatz dazu stellen Franz U. Pappi und seine Ko-Autoren fest, dass deutsche Parteien bei Koalitionsregierungen in den Bundesländern sich mitunter deutlich darin unterscheiden, in welchen Politikfeldern sie Ämter besetzen. ${ }^{12}$ Schließt man eine Zufälligkeit dieser Unterschiede aus, bleiben parteispezifisch unterschiedliche Bewertungen der Bedeutung der Ämter als einzige Erklärung.

In einem internationalen Vergleich ebenfalls zwölf westeuropäischer Demokratien testen Hanna Bäck, Marc Debus und Patrick Dumont, inwiefern derartige Unterschiede auf Ideologien zurückführbar sind, konkret, ob Parteien bestimmter Parteifamilien Portfolios systematisch häufiger besetzen als Parteien anderer Parteifamilien. ${ }^{13}$ Hierzu ordnen die Autoren einzelnen Parteifamilien Ministerien zu, bei denen sie eine besondere Affinität vermuten, zum Beispiel den Sozialisten das Portfolio Arbeit und den Liberalen das Wirtschaftsressort. Daneben stellen sie die Hypothese auf, dass die Häufigkeit der Nennung bestimmter Themen in Wahlprogrammen ein Indikator für die spätere Besetzung zugehöriger Ämter ist. Beide Hypothesen bestätigen sich, wobei die Erklärungskraft über die einzelnen Länder hinweg variiert.

Johannes Raabe untersucht den Prozess der Ämteraufteilung mit Hilfe von Divisorverfahren ${ }^{14}$, aufbauend auf einem Beitrag von Steven J. Brams und Todd R. Kaplan. ${ }^{15}$ Davon ausgehend, dass sich die Häufigkeit von Themennennungen in Wahlprogrammen als Maß

9 Vgl. ebenda.

10 Als problematisch erweisen sich vor allem unterschiedliche Zuschnitte von Ministerien und unvollständige Befragungen, so dass die Autoren eine Reihe zusätzlicher Annahmen treffen mussten, um ihre Analyse durchzuführen.

11 Paul V. Warwick / James N. Druckman, Portfolio Salience and the Proportionality of Payoffs in Coalition Governments, a.a.O. (Fn. 3), S. 648.

12 Vgl. Franz U. Pappi / Ralf Schmitt / Eric Linhart, Die Ministeriumsverteilung in den deutschen Landesregierungen seit dem Zweiten Weltkrieg, in: ZParl, 39. Jg. (2008), H. 2, S. 323 - 342.

13 Vgl. Hanna Bäck / Marc Debus / Patrick Dumont, Who Gets What in Coalition Governments? Predictors of Portfolio Allocation in Parliamentary Democracies, in: European Journal of Political Research, 50. Jg. (2011), H. 4, S. $441-478$.

14 Vgl. Johannes Raabe, Die Ministeriumsverteilung in Koalitionsregierungen - eine modelltheoretische Näherung mit Hilfe von Divisor-Verfahren, in: André Bächtiger / Susumu Shikano / Eric Linhart (Hrsg.), Jahrbuch für Handlungs- und Entscheidungstheorie, Band 8, Baden-Baden 2012 (im Erscheinen).

15 Vgl. Steven J. Brams / Todd R. Kaplan, Dividing the Indivisible: Procedures for Allocating Cabinet Ministries to Political Parties in a Parliamentary System, in: Journal of Theoretical Politics, 16. Jg. (2004), H. 2, S. $143-173$. 
nutzen lässt, um die Gewichtung von Interessen einzelner Parteien an unterschiedlichen Politikfeldern zu bestimmen, analysiert er, wie sich mit Hilfe solcher Divisorverfahren die letztendliche Ministerienverteilung erklären lässt. Auch Raabe kommt zu dem Schluss, dass die Berücksichtigung unterschiedlicher Interessenschwerpunkte der Parteien von Bedeutung ist, um die Prozesse der Ämteraufteilung zu erklären.

\section{Welche Parteien setzen welche Schwerpunkte bei der Ämterverteilung? Vorabinterviews und Fragebögen}

Festzuhalten bleibt also erstens, dass im Gegensatz zu quantitativen Fragen der Ämteraufteilung qualitative Aspekte mit wenigen Ausnahmen relativ unbeachtet blieben, hier also noch Forschungsbedarf besteht. Zweitens erhärten die bisherigen Ergebnisse die Vermutung, dass unterschiedliche Interessenschwerpunkte an einzelnen Ämtern seitens unterschiedlicher Parteien oder Parteifamilien existieren und sich diese auch auf den Prozess der Ämteraufteilung auswirken. Ordnet man den bisherigen Studien ihre genauen Ansatzpunkte zu (vgl. Abbildung 1), ist auch festzustellen, dass keine direkt bei der wichtigen unabhängigen Variablen, den unterschiedlichen Interessen (1), ansetzt, sondern entweder bei den Prozessen (2) oder bei den Ergebnissen der Ämterverteilung (3). Eine Ausnahme bildet lediglich der Beitrag von Laver / Hunt, der aber wiederum die wichtige parteispezifische Komponente völlig ausblendet. Eine systematische und direkte Beschäftigung mit der unabhängigen Variablen ist also dringend geboten, denn die Frage nach den Interessen von Parteien an unterschiedlichen Ämtern ist nicht nur für die Parteien selbst wichtig (etwa bei Fragen der Koalitionsbildung), sondern auch für deren Wähler.

Um zu ermitteln, welche Parteien schwerpunktmäßig an welchen Portfolios interessiert sind, wurden Parteivertreter befragt ${ }^{16}$, und zwar zunächst zwei Mitarbeiter der Staatskanzlei sowie die Parlamentarischen Geschäftsführer aller zu diesem Zeitpunkt im Landtag Schleswig-Holsteins vertretenen Fraktionen. ${ }^{17}$ Ziel dieser acht leitfadengestützten Vorabinterviews

16 Eine alternative Möglichkeit, diese Informationen zu beschaffen, wäre deren Ableitung aus anderen Daten, denen man eine hohe Korrelation mit den Interessenschwerpunkten der Parteien an Ämtern unterstellen kann (etwa Wahlprogrammen). Hanna Bäck / Marc Debus / Patrick Dumont (a.a.O., Fn. 13) haben einen signifikanten Einfluss der Nennhäufigkeit bestimmter Themen auf die Besetzung der entsprechenden Ämter festgestellt. Selbst unter Berücksichtigung weiterer Variablen liegt $M c F a d d e n s$ Pseudo $R^{2}$ in den Modellen von Bäck u.a. aber bei maximal 0,16. Würde man versuchen, die Ämteraufteilung mit Hilfe aller im Modell vorkommenden Variablen zu prognostizieren, läge der Anteil korrekter Vorhersagen in allen Modellvarianten bei unter 60 Prozent. Wahlprogramme sind also nur ein sehr grober Indikator und scheiden vorerst als Alternative aus.

17 Schleswig-Holstein eignete sich aus zwei Gründen besonders als Fall für die Vorabinterviews: Erstens waren zu diesem Zeitpunkt alle Parteien des seit 1990 auf Bundesebene existierenden Fünfparteiensystems (CDU/CSU, SPD, FDP, Grüne, PDS beziehungsweise Linke) in Fraktionsstärke im Landtag. Zudem konnte mit dem SSW zusätzlich eine Partei berücksichtigt werden, die rein landespolitisch von Bedeutung ist, und zwar über einen langen Zeitraum hinweg. Zweitens waren vergleichsweise viele der Landesparteien in unterschiedlichen Varianten an Koalitionsgesprächen beteiligt, so dass deren Vertreter fundierter Auskunft geben können als Funktionäre von Landesparteien, die sich in ständiger Opposition befinden. Vor der aktuell regierenden SchleswigHolstein-Ampel aus SPD, Grünen und SSW regierte eine schwarz-gelbe Koalition, davor eine Große Koalition. Innerhalb der letzten Jahre waren somit fünf der sechs befragten Parteien aktiv an Verhandlungen zur Regierungsbildung beteiligt. 


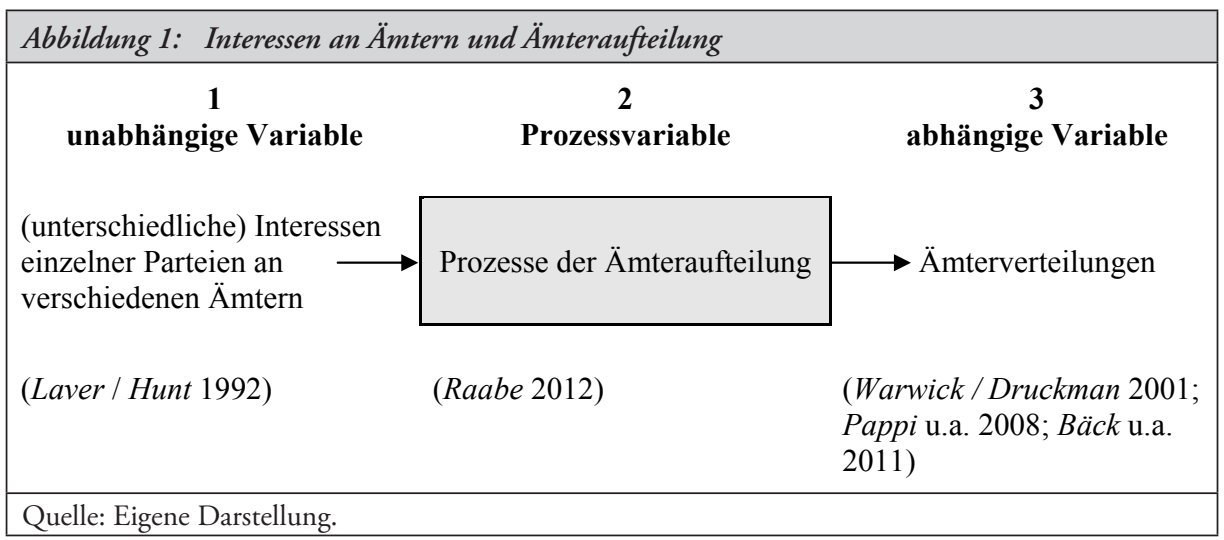

war neben der Prüfung und Feinabstimmung des Fragebogens für die schriftliche Befragung auch die Gewinnung von Hintergrundinformationen zur Präferenzbildung der Parteien sowie zur Ämtervergabe im Rahmen von Koalitionsverhandlungen. Aus den Interviews lassen sich für den an dieser Stelle ausschließlich betrachteten ersten dieser beiden Themenkomplexe drei wesentliche Ergebnisse festhalten, die für die weitere Untersuchung von Bedeutung sind:

(1) Die Relevanz eines Ressorts richtet sich in der Tat erstens nach den auf Landesebene angesiedelten Kompetenzen, aber auch nach den mit ihm verbundenen Querschnittsaufgaben (zum Beispiel durch Mitzeichnungsverfahren) und weiterhin, wie vermutet, nach den jeweiligen aktuellen Herausforderungen (beispielsweise Landesfinanzen). Die Gesprächspartner bestätigen damit in einem ersten Schritt, dass Portfolios in einer allgemeinen Bewertung unterschiedlich wichtig sind, dass das politische System beziehungsweise die politische Ebene eine Rolle spielen und dass die Bedeutung der Bereiche über die Zeit hinweg variieren kann. Im Hinblick auf parteispezifische Ressortinteressen wird neben inhaltlichen Schwerpunkten, ablesbar unter anderem am Wahlprogramm, auch das zur Verfügung stehende Personal als entscheidend für die Präferenzbildung benannt.

(2) Daraus ergibt sich bereits partiell die Schlussfolgerung, dass die Ressortpräferenzen der einzelnen Parteien keine festen Größen sind, sondern in Abhängigkeit von gesellschaftlichen Entwicklungen, Personen, Koalitionspartnern, Änderungen von Ressortzuschnitten und -zuständigkeiten, inneren Werten der Parteien und der Wählerzustimmung schwanken. Der „Kernbereich“ der einzelnen Parteien (in Bezug auf ihre Grundwerte, Schwerpunkte und Wählerschaft) wird aber durchgehend für sehr stabil gehalten. Trifft diese Experten-Einschätzung zu, hat das die erfreuliche Konsequenz, dass die Ergebnisse unserer Befragung mehr als eine Momentaufnahme darstellen und im Rahmen eines bestimmten Schwankungsbereichs für die befragten deutschen Parteien auf Landesebene längerfristige Gültigkeit besitzen.

(3) Die Frage der einheitlichen Bewertung der Ressorts innerhalb der Parteien und Fraktionen wird sehr unterschiedlich beantwortet: Das Spektrum reicht von der Vermutung einer weitgehenden Homogenität beziehungsweise Vorgabe der Präferenzen durch die Führung bis zu einer intensiven innerparteilichen Diskussion mit durchaus abweichenden Positionen. Die Behandlung von Parteien/Fraktionen als einheitliche Akteure stellt folglich eine (konzeptuell notwendige) Einschränkung der vorliegenden Untersuchung dar. Die wie auch immer zustande gekommene - von der Parteispitze vorgegebene oder innerparteilich ausge- 
handelte - Gesamtposition sollte sich jedoch in der Regel im Wahlprogramm spiegeln.

Im zweiten Schritt wurde mittels eines Fragebogens eine schriftliche Befragung der Fraktions- und Parteivorstände in allen Bundesländern durchgeführt. Hierbei wurden nur solche Vorstände angeschrieben, deren Partei zum jeweiligen Zeitpunkt in Fraktionsstärke im Landtag vertreten war. Der Erhebungszeitraum erstreckte sich von Januar bis Juni 2011. Fanden in dieser Zeit Landtagswahlen statt, wurde die Erhebung vor den jeweiligen Wahlen durchgeführt.

Nach einem knappen Anschreiben und einer kurzen Erklärung zum Zweck des Fragebogens wurde zunächst die Bedeutung der einzelnen Ministerien im Vergleich zu Ministerpräsident und Staatskanzlei abgefragt, wobei jeweils der zu dem Zeitpunkt aktuelle Zuschnitt der Ressorts in den einzelnen Bundesländern zugrunde gelegt wurde. Als Referenz wurde für den Ministerpräsidenten inklusive seiner Staatskanzlei der Wert 100 festgelegt. ${ }^{18}$

Um einen Vergleich mit dem Ministerpräsidenten und seiner Staatskanzlei ziehen zu können, war es notwendig, die Ministerien als Ganzes abzufragen. Durch das häufige Vorkommen mehrerer Geschäftsbereiche unter dem Dach eines Ministeriums und zudem durch die Unterschiedlichkeit der Zuschnitte im Ländervergleich ist es jedoch schwierig, herauszufiltern, ob ein bestimmter Geschäftsbereich zu einer hohen oder niedrigen Einschätzung der Bedeutung führt oder ob eine Kombination bestimmter beziehungsweise die Summe der Geschäftsbereiche ein Ministerium als wichtig erscheinen lassen. Um dies im Detail zu klären, wurden die Befragten zusätzlich gebeten, die Bedeutung der einzelnen Geschäftsbereiche innerhalb der Ministerien auf einer Siebener-Skala zu bewerten, und zwar zunächst aus einer allgemeinen, von spezifischen Parteiinteressen unabhängigen Perspektive und anschließend aus Sicht der eigenen Partei. ${ }^{19}$ Diese Unterscheidung ist notwendig, um herauszufinden, ob eine allgemeine Bedeutung überhaupt existiert, ob die Parteien die einzelnen Geschäftsbereiche also gleichermaßen als übermäßig wichtig oder unwichtig einschätzen. Außerdem kann mit dieser Zweiteilung dazwischen differenziert werden, ob Parteien ihrer eigenen Einschätzung nach auf einen bestimmten Geschäftsbereich besonders viel Gewicht legen und ob sie diesen im Vergleich mit den anderen Parteien tatsächlich als überdurchschnittlich bedeutsam bewerten.

Die Geschäftsbereiche wurden wie die Ministerien landesspezifisch aufgeführt, wobei jeweils die nominellen (in der Bezeichnung des Ministeriums aufgeführten) Bereiche berücksichtigt wurden. Dazu wurden weitere Geschäftsbereiche in einem Dreischritt ergänzt. Einige wurden aufgrund ihrer mutmaßlich hohen Bedeutung grundsätzlich abgefragt, sofern sie zumindest auf Abteilungsebene vertreten waren: Schule/Bildung (beziehungsweise Kultus), Forschung, Wissenschaft, Verbraucherschutz, Landwirtschaft, Arbeit, Soziales, Gesundheit, Frauen/Gleichstellung und Kinder/Jugend/Familie/Senioren. ${ }^{20}$ Zweitens wurden Bereiche speziell abgefragt, wenn sie auf Abteilungsebene vertreten waren, aber in einem Ministerium angesiedelt, das nicht das eigentliche Politikfeld dieser Abteilung abdeckte. So findet sich

18 Als Beispiel ist der hessische Fragebogen auf http://www.mzes.uni-mannheim.de/publications/ misc/linhart/2012zparl_Fragebogen.pdf hinterlegt. Der genaue Wortlaut der Fragestellung sowie der Aufbau des Fragebogens können dort eingesehen werden.

19 Siehe ebenda, Frage 3.

20 Weitere Geschäftsbereiche, die man als wichtig erachten könnte, wie Inneres, Justiz, Wirtschaft wurden stets auch im Namen eines Ministeriums auf Landesebene geführt, so dass auch diese Bereiche grundsätzlich immer abgefragt wurden. 
etwa die Abteilung für Reaktorsicherheit und Strahlenschutz in Schleswig-Holstein nicht im für Umwelt zuständigen Ministerium, sondern ist im Ministerium für Justiz, Gleichstellung und Integration angesiedelt. Drittens schließlich wurde auf mehrfachen Rat im Rahmen der Vorabinterviews die Aufgabe der Ressortkoordinierung mit abgefragt, für die im Wesentlichen die Staatskanzlei zuständig ist, ohne dies explizit in ihrem Namen zu führen.

\section{Rücklauf und Datenaufbereitung}

Insgesamt wurden 680 Personen aus 76 Landesverbänden befragt (siehe Tabelle 1). Die Union sowie die SPD waren in allen Landtagen in Fraktionsstärke vertreten, die FDP in 14, Grüne und Linke jeweils in 13. Hinzu kommen die NPD, die in zwei Landtagen vertreten war, sowie die Freien Wähler in Bayern und der SSW in Schleswig-Holstein. Da die Frak-

\begin{tabular}{|l|c|c|c|c|c|c|c|c|c|c|}
\hline Tabelle 1: Zurückgeschickte und versendete Fragebögen (in Klammern) \\
\hline Bundesland & $\begin{array}{c}\text { CDU/ } \\
\text { CSU }\end{array}$ & SPD & FDP & Grüne & Linke & NPD & FW & SSW & Summe & $\begin{array}{c}\text { Rück- } \\
\text { lauf- } \\
\text { quote } \\
\text { (in \%) }\end{array}$ \\
\hline $\begin{array}{l}\text { Baden- } \\
\text { Württemberg }\end{array}$ & $0(12)$ & $6(10)$ & $2(11)$ & $0(7)$ & - & - & - & - & $8(40)$ & 20,0 \\
Bayern & $2(14)$ & $3(12)$ & $4(10)$ & $1(7)$ & - & - & $3(8)$ & - & $13(51)$ & 25,5 \\
Berlin & $2(11)$ & $2(11)$ & $2(8)$ & $2(8)$ & $2(11)$ & - & - & - & $10(49)$ & 20,4 \\
Brandenburg & $3(8)$ & $2(10)$ & $3(7)$ & $1(4)$ & $2(8)$ & - & - & - & $11(37)$ & 29,7 \\
Bremen & $4(7)$ & $1(7)$ & - & $1(6)$ & $1(6)$ & - & - & - & $6(26)$ & 23,1 \\
Hamburg & $5(10)$ & $1(10)$ & - & $1(5)$ & $0(8)$ & - & - & - & $7(33)$ & 21,2 \\
Hessen & $3(12)$ & $4(14)$ & $2(8)$ & $3(6)$ & $3(8)$ & - & - & - & $15(48)$ & 31,3 \\
Mecklenburg- & $0(11)$ & $3(11)$ & $2(8)$ & - & $4(9)$ & $1(6)$ & - & - & $10(45)$ & 22,2 \\
Vorpommern & $4(14)$ & $9(12)$ & $2(8)$ & $1(9)$ & $1(7)$ & - & - & - & $17(50)$ & 34,0 \\
$\begin{array}{l}\text { Niedersachsen } \\
\text { Nordrhein- }\end{array}$ & $1(14)$ & $5(11)$ & $5(9)$ & $4(10)$ & $0(13)$ & - & - & - & $15(57)$ & 26,3 \\
Westfalen & $4(8)$ & $3(12)$ & $1(9)$ & - & - & - & - & - & $8(29)$ & 27,6 \\
Rheinland-Pfalz \\
Saarland
\end{tabular}


tions- und Parteivorstände der Landesparteien teilweise von sehr unterschiedlicher Größe sind, ergibt sich eine stark variierende Anzahl der Befragten nach Parteien (von sechs beim SSW über 90 bei den Grünen bis hin zu 174 Befragten bei CDU und CSU). Auch die Anzahl an Parteien in den Landtagen schwankt zwischen drei in Rheinland-Pfalz und sechs in Sachsen und Schleswig-Holstein. Demnach ist auch die Zahl der Befragten nach Bundesländern sehr unterschiedlich und bewegt sich zwischen 26 (Bremen mit vier Fraktionen und sehr kleinen Vorständen) und 57 (Nordrhein-Westfalen mit fünf Fraktionen und sehr großen Vorständen).

So ist es nicht allein auf unterschiedliche Rücklaufquoten zurückzuführen, dass 48 Fragebögen von SPD-Politikern in die Analyse eingehen, aber nur 23 von Grünen-Politikern und nur jeweils zwei beziehungsweise drei aus den Reihen von NPD, FW und SSW. Aufgrund der geringen Zahl an auswertbaren Fragebögen der Kleinstparteien NPD, FW und SSW und der damit verbundenen Schwierigkeit bei der Interpretation der Ergebnisse werden im Folgenden nur die fünf Parteien mit bundespolitischer Bedeutung (CDU und CSU zusammengefasst) diskutiert. ${ }^{21}$

Der Rücklauf über alle Parteien hinweg ist mit 27,2 Prozent insgesamt zufriedenstellend. Unter den Parteien mit bundespolitischer Bedeutung kam der anteilsmäßig größte Rücklauf von der FDP und der SPD, während CDU/CSU und Grüne zurückhaltender waren. Allerdings ist hier kein einheitlicher Trend innerhalb der Parteien über die Landesverbände hinweg festzustellen. So gab es einerseits auch SPD-Landesverbände komplett ohne Rücklauf (Sachsen-Anhalt), andererseits CDU-Landesverbände (Schleswig-Holstein: 60 Prozent) und Grünen-Landesverbände (ebenfalls Schleswig-Holstein: 57,1 Prozent) mit hohem Rücklauf. Es kann also nicht auf eine grundsätzlich geringere Antwortbereitschaft der Landesverbände einer Partei im Vergleich zu einer anderen geschlossen werden. Auch ein beidseitiger $t$-test ergibt, dass sich die Rücklaufquoten der einzelnen Parteien nicht signifikant voneinander unterscheiden.

Auch wenn in diesem Beitrag nicht die Analyse des Antwortverhaltens von Politikern im Detail behandelt wird, sei ein kleiner Exkurs hierzu erlaubt. Es darf vermutet werden, dass die Befragten unter gewissen Randbedingungen besonders zurückhaltend auf Fragen zu Koalitionen, speziell auf Fragen zur Ämteraufteilung in Koalitionen, reagieren. Eine solche Randbedingung kann eine anstehende Wahl oder eine schwierige Koalitionssituation sein. Insbesondere sollten Parteien sensibel reagieren, die in Regierungsverantwortung stehen und/oder sehr gute Chancen haben, nach einer bald anstehenden Wahl an der Regierung beteiligt zu sein. Eine lineare Regression auf die abhängige Variable „Rücklaufquote“ zeigt für alle drei Variablen „stattfindende Landtagswahl 2011“, „Regierungspartei“ und „ungewöhnliche Koalition“ negative Vorzeichen, wenngleich nur die beiden Variablen „stattfindende Landtagswahl 2011“ (auf 0,1 Prozent-Niveau) und „Regierungspartei“ (auf Fünfprozent-Niveau) statistisch signifikant sind. ${ }^{22}$

21 Für alle Interessierten stellen wir sämtliche Ergebnisse im Detail in einem Online-Anhang zur Verfügung, der auch die Ergebnisse zu NPD, FW und SSW enthält. Dieser Online-Anhang ist gemeint, wenn im Folgenden verkürzt auf „den Online-Anhang“ verwiesen wird. Er ist abrufbar unter http://www.mzes.uni-mannheim.de/publications/misc/linhart/2012zparl_Daten.xls.

22 Landtagswahlen fanden 2011 in Baden-Württemberg, Berlin, Bremen, Hamburg, MecklenburgVorpommern, Rheinland-Pfalz und Sachsen-Anhalt statt. Als Regierungsparteien wurden in jedem Bundesland die Parteien kodiert, die formal die Regierung stellt. Im Falle eines Regierungswechsels infolge einer Landtagswahl wurden sowohl die Parteien vor als auch nach dem Regierungswechsel 
Wichtig aber ist festzuhalten, dass aufgrund mehrerer Faktoren - unterschiedliche Größen der Partei- und Fraktionsvorstände, Vertretungshäufigkeiten der Parteien in den Landesparlamenten, Rücklaufquoten - die Menge verwertbarer Daten sowohl über die Parteien als auch über die Bundesländer hinweg deutlich variiert. So liegen für die Analyse neun Fragebögen der niedersächsischen SPD vor, aber nur einer der hamburgischen. Die schleswigholsteinische CDU geht mit sechs Fragebögen in die Untersuchung ein, der nordrheinwestfälische CDU-Landesverband nur mit einem. Ähnliches gilt, mal mehr, mal weniger, auch für die übrigen Parteien. Hierdurch entstehen Verzerrungen der Auswertungen sowohl über die Parteien als auch über die Landesverbände hinweg. Wenn etwa aus landesspezifischen Gründen ein bestimmter Geschäftsbereich in Sachsen als besonders bedeutsam angesehen wird, ein anderer in Sachsen-Anhalt, so würde die sächsische Einschätzung vier mal so stark in die Analyse eingehen wie die sachsen-anhaltische. Wir folgen daher der oben beschriebenen Sichtweise der Landesverbände als unitarische Akteure und ermitteln zunächst jeweils den Mittelwert aller Antwortenden für jedes Bundesland und jede Partei als gemeinsame Position des Landesverbands.

\section{Die Bedeutung einzelner Ministerien in den deutschen Bundesländern}

Die durchschnittliche Bedeutung der Ministerien im Vergleich untereinander, aber auch im Vergleich mit dem Ministerpräsidenten und seiner Staatskanzlei lässt sich nicht bundesweit, sondern nur für die jeweiligen Länder mit ihren speziellen Ressortzuschnitten vergleichen. Zwar gibt es Ministerien, die in jedem Bundesland identisch oder nahezu identisch auftauchen (etwa ein ausschließlich für Finanzen oder ausschließlich für Justiz zuständiges Ressort), die Mehrzahl besteht aber aus Kompositionen von Geschäftsbereichen, die landesspezifische Eigenheiten darstellen und nur sehr bedingt ihre Entsprechungen in anderen Bundesländern finden. ${ }^{23}$

Fixiert man - wie in unserem Fragebogen - die Bedeutung des Ministerpräsidenten mit seiner Staatskanzlei auf den Wert 100, wird die Bedeutung der Ministerien zwischen 41 und 107 eingeschätzt. Den höchsten Wert erzielt hierbei das Staatsministerium der Finanzen in Bayern, den niedrigsten das Ministerium für Gesundheit und Verbraucherschutz im Saarland. Tabelle 2 gibt einen Überblick über die Bewertung aller 133 in den Bundesländern vorkommenden Ministerien. ${ }^{24}$ Es zeigt sich, dass Ministerien grob als halb so wichtig bis

berücksichtigt. Die Variable „ungewöhnliche Koalition“ als Dummy-Variable bezieht sich auf die Bundesländer, in denen nach Meinung der Autoren unübliche Regierungsbündnisse im Jahr 2011 vorkamen/vorkommen. Dies sind die Jamaikakoalition im Saarland, die grün-rote Regierung in Baden-Württemberg und die Minderheitsregierung in Nordrhein-Westfahlen. Zusätzlich wurde Hamburg erfasst, da die Ende 2010 gescheiterte schwarz-grüne Koalition noch präsent gewesen sein dürfte. Die Vorzeichen und Signifikanzen gelten sowohl für bivariate als auch für multivariate Regressionen in verschiedenen Kombinationen, mit und ohne Berücksichtigung von Interaktionsvariablen.

23 So ist etwa der Geschäftsbereich Umwelt in Baden-Württemberg mit dem Geschäftsbereich Verkehr unter dem Dach eines Ministeriums untergebracht, in Bayern mit Gesundheit, in Hamburg mit Stadtentwicklung, in Mecklenburg-Vorpommern mit Verbraucherschutz und in Sachsen mit Landwirtschaft. Die einzelnen Umweltministerien der Länder sind also keineswegs vergleichbar.

24 Eine Liste aller Ministerien mit ihren durchschnittlichen Bewertungen findet sich im OnlineAnhang. 


\begin{tabular}{|l|c|c|c|c|c|c|c|c|}
\hline \multicolumn{7}{|l|}{ Tabelle 2: Verteilung der Bedeutsamkeitswerte über alle Ministerien } \\
\hline $\begin{array}{l}\text { durch- } \\
\text { schnittliche } \\
\text { Bedeutung }\end{array}$ & unter 50 & $\begin{array}{c}50 \text { bis } \\
\text { unter } 60\end{array}$ & $\begin{array}{c}60 \text { bis } \\
\text { unter } 70\end{array}$ & $\begin{array}{c}70 \text { bis } \\
\text { unter } 80\end{array}$ & $\begin{array}{c}80 \text { bis } \\
\text { unter } 90\end{array}$ & $\begin{array}{c}90 \text { bis } \\
\text { unter } 100\end{array}$ & $\begin{array}{c}100 \text { oder } \\
\text { größer }\end{array}$ & Summe \\
\hline $\begin{array}{l}\text { Anzahl } \\
\text { Anteil (in \%) }\end{array}$ & 2,26 & 17 & 29 & 42 & 26 & 14 & 2 & 133 \\
\hline
\end{tabular}

Fragebogen: Wenn Sie die Bedeutung des Ministerpräsidentenamtes samt Staatskanzlei gleich 100 setzen, wie schätzen Sie im Vergleich dazu die Bedeutung der einzelnen Ministerien ein?

Quelle: Eigene Erhebung.

gleich wichtig wie der Ministerpräsident und seine Staatskanzlei eingeschätzt werden. Der Mittelwert über alle Ressorts liegt bei 73,8 bei einer Varianz von 163,0. Ein hoher Anteil streut dabei um diesen Mittelwert und rangiert zwischen 70 und 80; nur vergleichsweise wenige Ministerien werden durchschnittlich als unter 60 Prozent so bedeutsam (rund 15 Prozent) oder über 90 Prozent so bedeutsam (rund 12 Prozent) wie der Ministerpräsident eingeschätzt.

Aus Sicht des Regierungschefs heißt das, dass sein Posten in der Regel als am wichtigsten angesehen wird, dass er aber nur in absoluten Ausnahmen für mehr als doppelt so wichtig gehalten wird wie ein Ministerium. Seine herausragende Stellung muss er sich meist mit mindestens einem Minister teilen, dem eine ähnlich hohe Bedeutung zugeschrieben wird (16 Werte über 90 bei 16 Bundesländern). Es ist auffällig, dass sich unter diesen 16 Ministerien mit sehr hoher Bedeutung zwölf Finanzressorts befinden. Bei den übrigen vier handelt es sich um das Staatsministerium für Wirtschaft, Arbeit und Verkehr in Sachsen, das Staatsministerium des Innern ebenfalls in Sachsen, das Ministerium des Innern und für Sport in Rheinland-Pfalz sowie das Staatsministerium für Unterricht und Kultus in Bayern.

Geht man die Reihenfolge der Bedeutung für die einzelnen Bundesländer durch, führt auch dabei in 15 von 16 Fällen das jeweilige Finanzministerium mit Werten zwischen 80 (Sachsen-Anhalt) und 107 (Bayern) die Liste an. Lediglich in Rheinland-Pfalz folgt es mit einem Wert von 90 dem Ministerium des Innern und für Sport (92) auf Rang 2. Derart eindeutige Ergebnisse finden sich für die folgenden Rangplätze nicht. Auffällig ist noch, dass das für Kultus beziehungsweise Bildung zuständige Ministerium in zehn der 16 Bundesländer als am zweitbedeutendsten angesehen wird; die Unterschiede zu den jeweiligen Ministerien auf Rang 3 sind jedoch schon deutlich geringer. Am Ende der Skala rangiert in neun der 16 Bundesländer das Justizressort.

Die Suche nach Unterschieden zwischen den Parteien ist an dieser Stelle noch nicht sonderlich ergiebig. Es zeigt sich aber schon hier, dass das Finanzministerium parteiübergreifend für besonders wichtig gehalten wird. Die Mehrheit der Partei- und Fraktionsvorstände aller Parteien weist diesem die jeweils höchsten Werte zu. Abweichungen für einzelne Landesverbände lassen sich dabei für alle Parteien finden. Für Union, SPD, FDP und Die Linke gilt, dass grob jeweils zwischen 80 und 85 Prozent ihrer antwortenden Landesverbände das Finanzministerium als am bedeutsamsten einschätzen - Rangplatzbindungen eingeschlossen. Für Bündnis 90/Die Grünen liegt dieser Wert deutlich niedriger, mit sechs von elf Landesverbänden aber immerhin noch über 50 Prozent. 


\section{Die Bewertung einzelner Geschäftsbereiche}

Die mitunter höchst unterschiedlichen Zuschnitte der Ministerien in den einzelnen Ländern erlauben es nicht, über die Bundesländer hinweg zu vergleichen und damit auch systematisch Unterschiede zwischen den einzelnen Parteien jenseits von Einzelfällen zu identifizieren. Aus diesem Grund enthält unser Fragebogen zusätzlich den oben beschriebenen Fragenkanon zu den einzelnen Geschäftsbereichen.

Befasst man sich zunächst mit der Einschätzung der allgemeinen Bedeutung der Geschäftsbereiche über alle Bundesländer und Parteien hinweg, erweisen sich die Geschäftsbereiche $^{25}$ Schule/Bildung, Finanzen, Energie, Inneres, Arbeit, Wirtschaft und Verkehr mit durchschnittlichen Bedeutsamkeitswerten über 5,0 als die wichtigsten, wobei sich Schule/ Bildung und Finanzen recht deutlich absetzen (siehe Tabelle 3). Ebenfalls über dem Durchschnittswert von 4,5 liegen die Geschäftsbereiche Soziales, Umwelt/Naturschutz, Wissenschaft und Kinder/Jugend/Familie/Senioren. Von leicht unterdurchschnittlicher allgemeiner Bedeutung sind Bau, Gesundheit, Landesplanung, Verbraucherschutz, Forschung, Integration, Technologie und Ernährung. Für am wenigsten bedeutsam mit Werten unter 4,0 werden die Geschäftsbereiche Kultur, Frauen/Gleichstellung, Landwirtschaft, Justiz, die Res-

\begin{tabular}{|l|c|l|c|}
\hline Tabelle 3: Allgemeine Bedeutung der Geschäftsbereiche \\
\hline Geschäftsbereich & $\begin{array}{r}\text { allgemeine } \\
\text { Bedeutung }\end{array}$ & \multicolumn{1}{|c|}{ Geschäftsbereich } & $\begin{array}{c}\text { allgemeine } \\
\text { Bedeutung }\end{array}$ \\
\hline Schule/Bildung & 6,0 & Landesplanung & 4,3 \\
Finanzen & 5,8 & Verbraucherschutz & 4,3 \\
Energie & 5,6 & Forschung & 4,3 \\
Inneres & 5,4 & Integration & 4,3 \\
Arbeit & 5,4 & Technologie & 4,1 \\
Wirtschaft & 5,4 & Ernährung & 4,1 \\
Verkehr & 5,0 & Kultur & 3,8 \\
Soziales & 4,7 & Frauen/Gleichstellung & 3,7 \\
Umwelt/Naturschutz & 4,7 & Landwirtschaft & 3,7 \\
Wissenschaft & 4,6 & Justiz & 3,7 \\
Kinder/Jugend/Familie/Senioren & 4,5 & Ressortkoordinierung & 3,5 \\
Durchschnitt & 4,5 & Sport & 3,5 \\
Bau & 4,4 & Bund und Europa & 3,5 \\
Gesundheit & 4,4 & Forsten & 3,1 \\
\hline Fragebogen: Gehen Sie bitte die Liste der Geschäftsbereiche in den einzelnen Ministerien durch und \\
schätzen Sie auf einer Siebener-Skala (1 = gering, 7= hoch) die Ihrer Meinung nach allgemeine Bedeutung \\
des jeweiligen Geschäftsbereichs unabhängig von speziellen Parteiinteressen. \\
Quelle: Eigene Erhebung.
\end{tabular}

25 Um die Tabellen nicht überzustrapazieren, weisen wir hier nur die Geschäftsbereiche aus, die in mindestens drei der 16 Bundesländer vorkommen. Es fehlen somit die Geschäftsbereiche Häfen, Infrastruktur, Innovation, ländliche Räume, Medien, Prävention, Reaktorsicherheit, Tourismus, Verfassung, Weinbau und Wohnen. Im Online-Anhang sind alle Geschäftsbereiche zu finden. Inhaltlich gleiche Geschäftsbereiche mit unterschiedlichen Namen wurden zuvor zusammengefasst, etwa Schule, Bildung und Kultus, die sich allesamt mit der Schulpolitik des jeweiligen Landes befassen. 
sortkoordinierung, Sport, Bundes- und Europaangelegenheiten sowie abgeschlagen der Bereich Forsten gehalten. Die niedrige Einschätzung der Ressortkoordinierung stellt dabei eine Diskrepanz zu den qualitativen Vorabinterviews dar, in denen auf die hohe Bedeutung der Ressortkoordinierung hingewiesen wurde.

Im Einklang mit den Ergebnissen des vorigen Abschnitts steht die große Wichtigkeit der Geschäftsbereiche Schule/Bildung und Finanzen. Ebenso spiegeln sich die häufig geringen Werte für die Justizministerien in einer vergleichsweise niedrigen Bedeutung des Geschäftsbereichs Justiz wider. Gleichzeitig ist aber auch feststellbar, dass die einzelnen Geschäftsbereiche unter dem Dach eines Ministeriums höchst divergierend eingeschätzt werden können und somit mutmaßlich in sehr unterschiedlicher Weise zu dessen Bedeutsamkeit insgesamt beitragen. Dies gilt nicht nur für Ministerien, die aus sehr verschiedenen Geschäftsbereichen zusammengesetzt sind, sondern auch für solche mit „üblichen“ Kombinationen.

Damit sind jene gemeint, die Geschäftsbereiche aus nur einem Politikfeld enthalten. Mit dieser Einteilung folgen wir Franz U. Pappi u.a., die solche Geschäftsbereiche zu gemeinsamen Politikfeldern zusammenfassen, die häufig unter dem Dach eines Ministeriums vereint sind und denen dadurch eine inhaltliche Nähe zugeschrieben wird. ${ }^{26}$ Auf diese Art und Weise identifizieren sie 14 Politikfelder $^{27}$, denen jeder vorkommende Geschäftsbereich zugeordnet werden kann. ${ }^{28}$

Tabelle 3 kann entnommen werden, dass auch einzelne Geschäftsbereiche aus ein und demselben Politikfeld mitunter als höchst unterschiedlich relevant angesehen werden. Besonders offensichtlich ist dies für das Politikfeld Kultus. Während Schule/Bildung die Liste anführt, wird Wissenschaft schon als erheblich weniger bedeutend erachtet. Die Geschäftsbereiche Forschung und Kultur werden bereits als unterdurchschnittlich wichtig angesehen; Sport liegt abgeschlagen auf dem drittletzten Rang. Ähnliches lässt sich für das Politikfeld Arbeit und Soziales feststellen: Die Geschäftsbereiche Arbeit und Soziales gelten als überdurchschnittlich bedeutsam, Kinder/Jugend/Familie/Senioren, Gesundheit und Integration als etwa durchschnittlich und Frauen/Gleichstellung als unterdurchschnittlich.

Versucht man später, die Wichtigkeit der Ministerien auf die der einzelnen Geschäftsbereiche herunter zu brechen, so ist also dringend zu beachten, dass hier deutliche Unterschiede bestehen können. Einige konkrete Ergebnisse mögen hierbei offensichtlich sein, andere nicht unbedingt. So wäre sicherlich auch ohne explizite Befragung zu erwarten gewesen, dass ein Ministerium für Inneres und Sport seine Bedeutung vor allem aufgrund des Geschäftsbereichs Inneres erlangt. Dass beim sächsischen Staatsministerium für Justiz und Europaangelegenheiten beide Geschäftsbereiche in etwa gleichermaßen (un)bedeutend sind, oder dass beim hessischen Ministerium der Justiz, für Integration und Europa der Bereich Integration gemeinhin als der wichtigste angesehen wird, wäre so nicht unbedingt zu erwarten gewesen.

26 Vgl. Franz U. Pappi / RalfSchmitt / Eric Linhart, a.a.O. (Fn. 12).

27 Diese 14 Politikfelder reduzieren sich für unsere Fragestellung auf zehn. Es entfallen die beiden Politikfelder Aufbau/Wiederaufbau und Kriegsfolgen, deren Geschäftsbereiche in aktuellen Ministerien nicht mehr auftauchen. Ebenso wenig treten bei uns Minister für Sonderaufgaben auf, so dass auch auf das Politikfeld Sonderaufgaben verzichtet wird. Der Ministerpräsident mit seiner Staatskanzlei wurde bei unserer Befragung vorgegeben und daher ebenfalls nicht in die weitere Auswertung einbezogen.

28 Siehe für die Zuordnung den Online-Anhang zum Beitrag von Franz U. Pappi / RalfSchmitt / Eric Linhart auf http://www.mzes.uni-mannheim.de/publications/misc/zparl08_ergaenzungstabelle. pdf. 
Zu erwähnen ist abschließend, dass die aus Tabelle 3 zu entnehmende Rangfolge der Bedeutung der Geschäftsbereiche weitgehend Konsens unter den Parteien ist. Erstellt man seperate Listen für die Antwortenden der einzelnen Parteien, bleiben die Reihenfolgen der Geschäftsbereiche im Großen und Ganzen gleich. Kleinere Unterschiede bestehen darin, dass beispielsweise die SPD und die Linke Finanzen an erste Stelle, Schule/Bildung dafür erst an zweite Stelle setzen, und dass aus Sicht der Grünen der Bereich Inneres eine höhere allgemeine Bedeutung hat als der Bereich Energie. Die ersten sieben der Liste in Tabelle 3, also die Geschäftsbereiche mit einem Durchschnittswert über 5,0, machen auch für jede einzelne Partei die Top 7 aus - Ausnahme ist die Union, zu deren internen Top 7 Soziales anstatt Verkehr zählt. ${ }^{29}$ Im mittleren und unteren Bereich kommen mitunter größere Abweichungen vor; insgesamt kann aber durchaus davon ausgegangen werden, dass die einzelnen Parteien die allgemeine Bedeutung der Geschäftsbereiche sehr ähnlich einschätzen - trotz unterschiedlicher Interessenschwerpunkte aufgrund ihrer Programmatik.

\section{Schwerpunkte der einzelnen Parteien - absolut und relativ}

Befragt man die Vorstände hingegen nach der Bedeutung der Geschäftsbereiche bewusst aus Sicht der Parteien, können Schwerpunktsetzungen jenseits dieser Ähnlichkeit identifiziert werden. Hierbei sind zwei Fragen von besonderem Interesse: Erstens, welchen Geschäftsbereichen weisen die jeweiligen Parteien absolut gesehen eine ausnehmend große Wichtigkeit zu? Zweitens, bei welchen Geschäftsbereichen lässt sich eine besonders hohe Diskrepanz zwischen der allgemein zugewiesenen Bedeutung und der parteispezifischen identifizieren?

Zur Beantwortung der ersten Frage wurden direkt die Antworten des Fragebogens (Frage 3, rechte Spalte) ausgewertet. Die in Tabelle 4 gezeigten Ergebnisse sind die Mittelwerte über die jeweiligen Landesverbände, jeweils für die einzelnen Parteien separat ausgewiesen. Dargestellt sind die sieben Geschäftsbereiche, die die Parteien als die wichtigsten eingeschätzten. ${ }^{30}$

Die Geschäftsbereiche, die allgemein für bedeutend gehalten werden, werden häufig auch als bedeutsam aus Sicht der Parteien genannt. So sind die drei wichtigsten Geschäftsbereiche der allgemeinen Einschätzung (Schule/Bildung, Finanzen, Energie) auch jeweils in den Top 7 der einzelnen Parteien vertreten. Im Detail sind jedoch Unterschiede erkennbar. Während der Bereich Finanzen von Union und FDP besonders herausgehoben wird, taucht er bei Grünen und Linken erst an sechster beziehungsweise siebter Stelle auf.

Bei CDU und CSU fällt auf, dass Inneres und Wirtschaft ausnehmend hoch bewertet werden, insgesamt aber die Liste der ersten sieben exakt mit den ersten sieben der allgemeinen Bedeutung aller Parteien (siehe Tabelle 3) übereinstimmt. SPD und Linke haben gemein, dass Schule/Bildung und Arbeit als besonders wichtig angesehen werden. Anstatt der Geschäftsbereiche Inneres und Verkehr finden sich in den Listen ihrer Top 7 Soziales und Integration. Die FDP setzt vor allem auf Finanzen und Wirtschaft, nennt aber im Gegensatz zu den anderen Parteien auch Wissenschaft und Forschung als eigene Schwerpunkte. Relativ stark setzen sich die Grünen mit eigenen Schwerpunkten von der allgemeinen Liste der ersten sieben ab: Zwar nennen auch sie die Themen Energie, Schule/Bildung und Finanzen,

29 Siehe im Detail den Online-Anhang zu diesem Beitrag.

30 Eine vollständige Liste der Werte für alle Geschäftsbereiche findet sich ebenfalls im Online-Anhang. 


\begin{tabular}{|c|c|c|c|c|c|c|c|c|c|}
\hline \multicolumn{2}{|c|}{$\mathrm{CDU} / \mathrm{CSU}$} & \multicolumn{2}{|r|}{ SPD } & \multicolumn{2}{|r|}{ FDP } & \multicolumn{2}{|r|}{ Grüne } & \multicolumn{2}{|r|}{ Linke } \\
\hline 6,7 & Finanzen & 6,8 & $\begin{array}{l}\text { Schule/ } \\
\text { Bildung }\end{array}$ & 6,8 & Finanzen & 7,0 & Energie & 6,6 & Arbeit \\
\hline 6,4 & Inneres & 6,7 & Arbeit & 6,7 & Wirtschaft & 6,4 & $\begin{array}{l}\text { Umwelt/ } \\
\text { Natur }\end{array}$ & 6,5 & $\begin{array}{l}\text { Schule/ } \\
\text { Bildung }\end{array}$ \\
\hline 6,4 & Wirtschaft & 6,4 & Energie & 6,5 & $\begin{array}{l}\text { Schule/ } \\
\text { Bildung }\end{array}$ & 6,4 & Integration & 6,5 & Energie \\
\hline 6,2 & $\begin{array}{l}\text { Schule/ } \\
\text { Bildung }\end{array}$ & 6,3 & Soziales & 6,3 & Wissenschaft & 6,2 & $\begin{array}{l}\text { Schule/ } \\
\text { Bildung }\end{array}$ & 6,5 & Soziales \\
\hline 6,2 & Energie & 6,2 & Finanzen & 6,1 & Energie & 6,1 & Verbr.-Schutz & 6,3 & Frauen \\
\hline 5,8 & Verkehr & 6,1 & Integration & 6,0 & Forschung & 5,8 & Finanzen & 6,3 & Integration \\
\hline 5,7 & Arbeit & 5,8 & Wirtschaft & 6,0 & Arbeit & 5,6 & Frauen & 6,0 & Finanzen \\
\hline
\end{tabular}

Fragebogen: Gehen Sie bitte die Liste der Geschäftsbereiche in den einzelnen Ministerien durch und schätzen Sie auf einer Siebener-Skala ( 1 = gering, 7 = hoch) die Bedeutung des jeweiligen Geschäftsbereichs speziell aus Sicht Ihrer Partei.

Anmerkung: Frauen $=$ Frauen/Gleichstellung; Verbr. - Schutz $=$ Verbraucherschutz .

Quelle: Eigene Erhebung.

setzen ansonsten aber auf Umwelt/Natur, Integration, Verbraucherschutz und - sonst eher als wenig bedeutsam eingeschätzt - Frauen/Gleichstellung. Insgesamt zeigt sich somit zum einen eine Konstanz in der Einschätzung besonders wichtiger Geschäftsbereiche, zum anderen sind aber durchaus Schwerpunktsetzungen der einzelnen Parteien erkennbar.

Um dieser Schwerpunktsetzung verstärkt nachzugehen, ist zu untersuchen, wo die Parteienvertreter die größten Unterschiede zwischen allgemeiner Einschätzung und Bedeutung aus Sicht ihrer eigenen Parteien vermuten. Es geht also um die durchschnittlichen Differenzen dessen, was die Parteivertreter in Frage 3 des Fragebogens links und rechts angekreuzt haben. Tabelle 5 gibt für diese Frage die Top 7 an, die vollständige Tabelle befindet sich im Online-Anhang.

Die Befragten attestieren ihren Parteien generell ein überdurchschnittlich hohes Interesse an allen Geschäftsbereichen. Bildet man die Mittelwerte über die allgemeine und die parteispezifische Bedeutung, so liegt letzterer mit 5,1 um rund 0,6 über der allgemeinen. Zwischen allgemeiner und parteispezifischer Bewertung gibt es also eine gewisse Schieflage, die um den Differenzwert 0,6 zu korrigieren ist, oder - mit anderen Worten: Gibt eine Partei an, einen Geschäftsbereich um 0,6 wichtiger zu bewerten als die allgemeine Bedeutung, so bewertet sie diesen letztendlich exakt durchschnittlich.

Davon unbenommen zeigt die Tabelle diejenigen Geschäftsbereiche, die die Parteivertreter als herausragend im Vergleich zur allgemeinen Bedeutung sehen. Die absolute Wichtigkeit kann hierbei sehr unterschiedlich sein. So ist die CDU/CSU nach Eigeneinschätzung etwa gleichermaßen überdurchschnittlich engagiert in den Bereichen Forsten und Finanzen; absolut gesehen hält sie den Geschäftsbereich Finanzen aber für den bedeutendsten überhaupt $(6,7)$, während Forsten im unteren Drittel rangiert $(4,3)$. Ein hoher Wert der CDU/ CSU in Tabelle 5 zum Beispiel für Landwirtschaft bedeutet also nicht, dass die Union diesen Geschäftsbereich für besonders wichtig erachtet, sondern dass sie glaubt, diesen Geschäftsbereich im Vergleich zu den anderen Parteien als besonders wichtig zu erachten. Man könnte die in Tabelle 5 aufgelisteten Geschäftsbereiche somit als komparative oder relative Schwerpunkte der Parteien interpretieren. 


\begin{tabular}{|c|c|c|c|c|c|c|c|c|c|}
\hline \multicolumn{10}{|c|}{$\begin{array}{l}\text { Tabelle 5: Die sieben Geschäftsbereiche mit den stärksten Unterschieden zwischen allgemeiner } \\
\text { und parteispezifischer Bedeutung }\end{array}$} \\
\hline \multicolumn{2}{|c|}{$\mathrm{CDU} / \mathrm{CSU}$} & \multicolumn{2}{|r|}{ SPD } & \multicolumn{2}{|r|}{ FDP } & \multicolumn{2}{|r|}{ Grüne } & \multicolumn{2}{|r|}{ Linke } \\
\hline 1,4 & Forsten & 1,7 & Soziales & 1,7 & Forschung & 2,3 & Integration & 2,0 & Forsten \\
\hline 1,3 & $\begin{array}{l}\text { Landwirt- } \\
\text { schaft }\end{array}$ & 1,6 & Arbeit & 1,4 & $\begin{array}{l}\text { Wissen- } \\
\text { schaft }\end{array}$ & 2,3 & $\begin{array}{l}\text { Umwelt/ } \\
\text { Natur }\end{array}$ & 1,9 & Frauen \\
\hline 1,3 & Finanzen & 1,6 & Frauen & 1,3 & Wirtschaft & 2,1 & Verbr.-Schutz & 1,7 & Soziales \\
\hline 1,1 & $\begin{array}{l}\text { Ressort- } \\
\text { koordination }\end{array}$ & 1,3 & Integration & 1,2 & Finanzen & 2,1 & Frauen & 1,7 & Integration \\
\hline 1,1 & Wirtschaft & 1,0 & Energie & 1,0 & Justiz & 1,7 & Energie & 1,5 & Energie \\
\hline 0,9 & Bau & 0,9 & KJFS & 0,8 & $\begin{array}{l}\text { Landwirt- } \\
\text { schaft }\end{array}$ & 1,7 & Bund/Europa & 1,2 & Arbeit \\
\hline 0,9 & Inneres & 0,9 & Technologie & 0,8 & Kultur & 1,5 & Kultur & 1,1 & Verbr.-Schutz \\
\hline & $\begin{array}{l}\text { kung: Zu } \\
\text { e/Seniore }\end{array}$ & & vgl. Tabelle & & Frauen = & $\mathrm{n} / \mathrm{s}$ & chstellu & & $\begin{array}{l}\text { Kinder/Jugend/ } \\
\text { utz. }\end{array}$ \\
\hline
\end{tabular}

Die Daten der Tabelle 5 bestätigen einige bereits in Tabelle 4 identifizierte Schwerpunkte, etwa die Bereiche Umwelt/Natur, Verbraucherschutz und Frauen/Gleichstellung bei den Grünen, Forschung und Wissenschaft bei der FDP sowie Inneres bei der CDU/CSU. Auch Geschäftsbereiche, die absolut gesehen für besonders wichtig gehalten werden, tauchen zugleich als relative Schwerpunkte auf. Zu nennen sind hier Wirtschaft und Finanzen bei Union und FDP, Arbeit bei SPD und Linken sowie Energie bei SPD, Grünen und Linken. Darüber hinaus lassen sich in Tabelle 5 Geschäftsbereiche identifizieren, die absolut gesehen nicht zu den bedeutsamsten gerechnet werden, bei denen die Parteien auf niedrigerem Niveau aber eine vergleichsweise hohe parteispezifische Relevanz benennen. Solche Geschäftsbereiche sind bei der CDU/CSU Forsten, Landwirtschaft, die Ressortkoordinierung und Bau, bei der SPD Soziales, Frauen/Gleichstellung, Integration, Kinder/Jugend/Familie/Senioren und Technologie, bei der FDP neben den oben schon genannten Justiz, Landwirtschaft und Kultur, bei den Grünen Integration, Bundes- und Europaangelegenheiten sowie Kultur und bei der Linken Forsten, Frauen/Gleichstellung, Soziales, Integration und Verbraucherschutz. Gerade mithilfe der komparativen Schwerpunkte lässt sich erklären, weshalb Parteien überdurchschnittlich häufig Ministerien in Politikfeldern besetzen, deren Geschäftsbereiche sie absolut gesehen nicht als ausnehmend wichtig einschätzen, etwa die Union im Bereich Landwirtschaft oder die FDP im Bereich Justiz. ${ }^{31}$

\section{Der Versuch, Vergleichbarkeit zu schaffen: die Bedeutung von Politikfeldern}

Will man systematisch zwischen Parteien über die Landesverbände hinweg vergleichen, eignen sich die Ministerien als Basis nicht. Wie oben dargelegt gibt es zwar Ressorts, die in sehr ähnlicher Form in jedem Bundesland vorkommen, die Mehrzahl besteht aber aus jeweils eigenen Zusammenstellungen von Geschäftsbereichen. Auf dieser Ebene ist zwar Vergleich-

31 Vgl. Franz U. Pappi / Ralf Schmitt / Eric Linhart, a.a.O. (Fn. 12), S. 333. 
barkeit gegeben; sie erscheint aber als zu feingliedrig. Für Spezialfragen mag es von Interesse sein, ob eine Partei einen stärkeren Schwerpunkt auf den Geschäftsbereich Forschung legt, während eine andere Wissenschaft für bedeutsamer hält. Zunächst ist es aber wichtiger, die Parteischwerpunkte größerer inhaltlicher Bereiche zu erfassen, wofür die Ebene der Politikfelder geeignet ist. ${ }^{32}$

Die von Pappi u.a. identifizierten Politikfelder resultieren gerade daraus, welche Geschäftsbereiche häufig unter dem Dach desselben Ministeriums vereint sind beziehungsweise waren. Für die Zuschnitte der Ressorts im Befragungszeitraum bestätigen sich diese Verknüpfungen. In Thüringen etwa trifft dies für das Ministerium für Bildung, Wissenschaft und Kultur (Politikfeld: Kultus) oder das Ministerium für Soziales, Familie und Gesundheit (Politikfeld: Arbeit und Soziales) zu. Trotz mehrerer Geschäftsbereiche ist die Zuordnung zu einem speziellen Politikfeld also eindeutig. Es gibt aber auch Ministerien mit Geschäftsbereichen aus unterschiedlichen Politikfeldern, zum Beispiel ebenfalls in Thüringen das Ministerium für Wirtschaft, Arbeit und Technologie (Politikfelder: Wirtschaft und Verkehr; Arbeit und Soziales) oder das Ministerium für Landwirtschaft, Forsten, Umwelt und Naturschutz (Politikfelder: Landwirtschaft; Umwelt und Landesplanung). Umgekehrt zeigen die Beispiele, dass sich auch einzelne Politikfelder auf verschiedene Ministerien verteilen können, wie hier bei Arbeit und Soziales erkennbar. Die Gewichtungen der einzelnen Politikfelder durch die Befragten sind also nicht direkt an den Gewichtungen für die Ministerien ablesbar. Es muss eine Methode gefunden werden, um anhand der den Ministerien zugewiesenen Werte die Bedeutung der einzelnen Politikfelder abzuschätzen. Hierbei stellt es eine Herausforderung dar, die Methode so zu wählen, dass die Ergebnisse sowohl mit den Antworten auf die Frage nach der Wichtigkeit der Ministerien als auch der einzelnen Geschäftsbereiche konsistent sind. Gleichzeitig ist diese Herausforderung eine Chance, die Methode intern zu validieren.

Als valide hat sich eine Methode herausgestellt, die den Bereich Finanzen als Anker nutzt. Dieser eignet sich deshalb optimal, weil er in jedem Bundesland auftaucht und in der Regel sowohl alleine das Finanzministerium definiert als auch einziger Geschäftsbereich im Politikfeld Finanzen ist. Bewertungen von Geschäftsbereich, Ministerium und Politikfeld können somit gleichgesetzt werden und bilden die Grundlage für weitere Abschätzungen. In einem zweiten Schritt ergeben sich daraus die Bedeutsamkeitswerte der weiteren Geschäftsbereiche. Wird dem Finanzministerium beispielsweise eine Bedeutung von 90 zugewiesen, so weisen wir auch dem Geschäftsbereich Finanzen diesen Wert zu. Die weiteren Abschätzungen ergeben sich aus den relativen Bewertungen der einzelnen Geschäftsbereiche. Wurden Finanzen auf der Skala mit sechs bewertet, der Bereich Justiz mit vier, so weisen wir dem Geschäftsbereich Justiz eine Bedeutsamkeit von 60 zu (vier Sechstel mal der Bedeutsamkeit von Finanzen). ${ }^{33}$

Die Bedeutsamkeit eines Ministeriums setzt sich dann zusammen aus den Bedeutsamkeiten der einzelnen Geschäftsbereiche, die es enthält. Ein einfaches Aufsummieren der entsprechenden Werte würde allerdings zu kurz greifen. Vielmehr scheint sich die Bedeutung eines Ministeriums am Mittelwert seiner Geschäftsbereiche beziehungsweise am Wert des wertvollsten Geschäftsbereichs zu orientieren - wobei das erstgenannte Vorgehen die Wich-

32 Vgl. ebenda.

33 Formal ausgedrückt: Bezeichnet $y_{F}$ den Bedeutsamkeitswert des Finanzministeriums, $z_{F}$ den Skalenwert für den Geschäftsbereich Finanzen und $z_{i}$ den Skalenwert für einen weiteren Geschäftsbereich $i$, so ergibt sich der Bedeutsamkeitswert für $i$ als $y_{i}=y_{F} z_{i} / z_{F}$. 


\begin{tabular}{|c|c|c|c|c|c|c|}
\hline & alle & CDU/CSU & SPD & FDP & Grüne & Linke \\
\hline Arbeit und Soziales & 87,9 & 75,3 & 96,2 & 75,8 & 86,6 & 97,9 \\
\hline $\begin{array}{l}\text { Aufbau und Wieder- } \\
\text { aufbau }\end{array}$ & - & - & - & - & - & - \\
\hline Bau & 71,2 & 70,8 & 73,5 & 65,5 & 73,2 & 66,3 \\
\hline Kultus & 87,2 & 79,0 & 91,2 & 86,4 & 86,8 & 91,4 \\
\hline Bund und Europa & 64,0 & 58,5 & 62,3 & 61,6 & 74,7 & 59,6 \\
\hline Kriegsfolgen & - & - & - & - & - & - \\
\hline Landwirtschaft & 73,8 & 66,7 & 68,0 & 69,5 & 89,0 & 74,5 \\
\hline Finanzen & 93,0 & 92,4 & 94,0 & 96,4 & 85,4 & 91,2 \\
\hline Justiz & 62,1 & 59,2 & 54,1 & 69,2 & 62,0 & 62,0 \\
\hline Inneres & 81,3 & 89,5 & 83,9 & 80,4 & 69,0 & 69,9 \\
\hline $\begin{array}{l}\text { Umwelt und Landes- } \\
\text { planung }\end{array}$ & 80,6 & 68,8 & 82,7 & 68,9 & 93,8 & 85,7 \\
\hline Wirtschaft und Verkehr & 88,7 & 85,9 & 88,6 & 91,1 & 85,7 & 87,4 \\
\hline
\end{tabular}

tigkeit der Politikfelder eher unter-, die zweite sie eher überschätzt. Der Durchschnitt beider Aggregationsmechanismen trifft die tatsächliche Einschätzung ziemlich genau. ${ }^{34}$ Davon ausgehend, dass es sich bei der Aggregation von Geschäftsbereichen zu Politikfeldern ähnlich verhält wie zu Ministerien, zeigt Tabelle 6 die entsprechend geschätzten Werte. ${ }^{35}$

Die Tabellenwerte sind nur über die Politikfelder, nicht aber über die Parteien hinweg vergleichbar, da sie jeweils für sich genommen den Bezugspunkt des Ministerpräsidenten und seiner Staatskanzlei (gleich 100) besitzen. Ein im Vergleich zur CDU/CSU höherer Wert der SPD beim Politikfeld Finanzen heißt also nicht, dass die SPD dieses Politikfeld für wichtiger hält als die Union. Er bedeutet zunächst nur, dass ihr dieses Politikfeld im Vergleich zum Ministerpräsidenten wichtiger ist. Generell hohe (niedrige) Werte einer Partei lassen also eher auf eine eingeschätzte relative Schwäche (Stärke) des Ministerpräsidenten rückschließen als auf ein allgemein hohes (niedriges) Interesse an allen Politikfeldern.

Betrachtet man weiter die gemeinsame Einschätzung aller Parteien, ragt das Politikfeld Finanzen mit dem Höchstwert über 90 hinaus. Dieser hohe Wert resultiert unmittelbar aus dem Geschäftsbereich Finanzen, der als ausnehmend bedeutend gilt (vgl. Tabelle 3) und faktisch allein das gleichnamige Politikfeld prägt. Es folgen mit Wirtschaft/Verkehr, Arbeit/ Soziales und Kultus drei Politikfelder, deren einzelne Geschäftsbereiche sehr unterschiedlich beurteilt werden. So finden sich dort sowohl Geschäftsbereiche, die meist für sehr wichtig gehalten werden (Energie; Arbeit; Schule/Bildung), als auch solche, denen eine eher geringe

34 Vergleicht man die tatsächlich genannten Bedeutsamkeitswerte einzelner Ministerien mit den geschätzten, die sich durch Aufsummieren der Werte der Geschäftsbereiche errechnen lassen, so ergibt sich ein durchschnittlicher Fehler von rund 125, der dieses Vorgehen als ungeeignet enttarnt. Die Orientierung am Maximalwert oder am Mittelwert der vorkommenden Geschäftsbereiche führt hingegen zu akzeptablen Abweichungen von 16 beziehungsweise 12 Einheiten.

35 Tabelle 6 zeigt die jeweiligen Werte, die aus dem Durchschnitt der Methoden ,Maximum' und ,Mittelwert' resultieren. Im Online-Anhang finden sich zudem die Bedeutsamkeitswerte für die einzelnen Geschäftsbereiche sowie der Methoden ,Maximum’ und ,Mittelwert' separat. 
Bedeutung zugesprochen wird (Technologie; Frauen/Gleichstellung; Kultur). Diese drei Politikfelder erreichen hohe Werte knapp unter 90, da sie mindestens einen als sehr relevant angegebenen Geschäftsbereich enthalten (die eben genannten) und darüber hinaus mindestens einen weiteren überdurchschnittlich bewerteten (Wirtschaft; Soziales; Wissenschaft). Mit Werten knapp über 80 werden Inneres sowie Umwelt und Landesplanung als ungefähr gleich bedeutsam eingeschätzt, wobei im Politikfeld Umwelt und Landesplanung der Geschäftsbereich Umwelt/Naturschutz herausragt. Im Politikfeld Inneres ist nahezu ausschließlich der gleichnamige Geschäftsbereich zu finden. Mit Werten zwischen 70 und 75 folgen die beiden Politikfelder Landwirtschaft und Bau. Letzteres ist wieder ein Politikfeld, das im Wesentlichen mit dem gleichnamigen Geschäftsbereich gleichgesetzt werden kann; im Politikfeld Landwirtschaft tauchen vor allem die Geschäftsbereiche Landwirtschaft und Verbraucherschutz auf, wobei der Verbraucherschutz insgesamt als bedeutsamer angesehen wird. Am Ende der Skala rangieren mit Bund/Europa und Justiz (Werte zwischen 60 und 65) wieder zwei Politikfelder, die im Wesentlichen aus jeweils einem Geschäftsbereich bestehen.

Blickt man auf die einzelnen Parteien, sind sowohl Gemeinsamkeiten als auch Unterschiede feststellbar. Gemeinsamkeiten bestehen etwa darin, dass alle Parteien mit Ausnahme der Grünen das Politikfeld Finanzen als das wichtigste ansehen. Auch Kultus (Ausnahme: CDU/CSU) und Wirtschaft/Verkehr zählen jeweils zu den bedeutenderen Politikfeldern, während die Politikfelder Bund/Europa (Ausnahme: Grüne) und Justiz (Ausnahme: FDP) auch bei den einzelnen Parteien am Ende der Skala rangieren. Ein auffälliger Unterschied zwischen den Parteien findet sich beim Politikfeld Arbeit und Soziales, das SPD und Linke als das wichtigste angeben, während es bei Union und FDP nur einen Platz im Mittelfeld einnimmt.

Geht man auf die Parteien im Einzelnen ein, so ist für die Union anzumerken, dass von ihren Vertretern das Politikfeld Inneres als ausnehmend bedeutsam angegeben wird. Neben Kultus und Arbeit/Soziales wird auch Umwelt/Landesplanung nur unterdurchschnittlich bewertet. Der etwas unterdurchschnittliche Wert beim Politikfeld Landwirtschaft resultiert vor allem aus einer sehr niedrigen Einschätzung des zugehörigen Geschäftsbereichs Verbraucherschutz, während der Geschäftsbereich Landwirtschaft selbst als überdurchschnittlich wichtig angesehen wird.

SPD und Linke fallen wie bereits erwähnt durch vergleichsweise hohe Einschätzungen der Bedeutung der Politikfelder Arbeit/Soziales und Kultus auf. Unterdurchschnittlich werden bei den Sozialdemokraten die Politikfelder Landwirtschaft und Justiz bewertet, bei den Linken Bau und Inneres.

Die FDP besitzt neben Finanzen nur einen weiteren herausragenden Schwerpunkt: Wirtschaft und Verkehr. Neben Arbeit/Soziales wird vor allem auch das Politikfeld Umwelt/ Landesplanung von ihren Befragten als unterdurchschnittlich relevant angesehen. Es ist auffällig, dass die FDP, wenn auch auf insgesamt niedrigem Niveau, das Politikfeld Justiz relativ besser bewertet als die anderen Parteien. Bei ihr rangiert es nicht als Schlusslicht, sondern immerhin noch vor Umwelt/Landesplanung, Bau und Bund/Europa.

Die Grünen besitzen ein Profil, das am stärksten von den übrigen Parteien abweicht. Nicht überraschend ist die Einschätzung des Politikfelds Umwelt/Landesplanung an erster Stelle. Es folgen mit etwa gleich großen Werten die Politikfelder Landwirtschaft ${ }^{36}$, Kultus,

36 Die Grünen schätzen beide zentralen Geschäftsbereiche im Politikfeld Landwirtschaft als überdurchschnittlich bedeutsam ein, stärker noch den Verbraucherschutz. 
Arbeit/Soziales, Wirtschaft/Verkehr und - erst auf dem sechsten Rang - Finanzen. Im unteren Bereich fällt auf, dass Bund und Europa als wichtiger eingeschätzt wird als Inneres, was sowohl auf einen relativ hohen Wert von Bund/Europa als auch auf einen sehr niedrigen für Inneres zurückzuführen ist.

\section{Grundlage für die Analyse von Ämteraufteilungen, Erkenntnisse für die Bildung von Koalitionen}

Die Aufteilung der Ministerien ist aus qualitativer Sicht nicht hinreichend erforscht. Um die beobachtete Verteilung der Ressorts erklären zu können, ist es notwendig, zunächst die Einschätzungen ihrer Wichtigkeit durch die verschiedenen Parteien zu kennen, denn die realen Ministerien sind die Einheiten, die dem Ministerpräsidenten und seiner Staatskanzlei gegenüber stehen und daher am ehesten geeignet sind, in Relation zu diesem bewertet zu werden. Gleichwohl sind die Ministerien in den einzelnen Ländern so unterschiedlich zusammengesetzt, dass die Ebene der Ministerien allein unzureichend ist, um die Ämterallokation zu erklären. Die Ebene der einzelnen Geschäftsbereiche ist dafür geeigneter. Hier lässt sich feststellen, dass parteiübergreifend ein Konsens besteht über bestimmte Geschäftsbereiche, die als allgemein besonders bedeutend (Schule/Bildung, Finanzen, Energie) oder weniger bedeutend (Sport, Bund/Europa, Forsten) angesehen werden. Darüber hinaus lassen sich parteispezifische Interessen an bestimmten Geschäftsbereichen identifizieren.

Auch für die Politikfelder zeigen sich neben gemeinsamen Einschätzungen aller Parteien (hohe Bedeutung von Finanzen sowie Wirtschaft und Verkehr, niedrige Bedeutung von Bund und Europa sowie Justiz) Schwerpunkte beziehungsweise relative Schwerpunkte einzelner Parteien. Neben zu erwartenden Ergebnissen wie einer Affinität der Grünen zu Umwelt/Landesplanung oder von SPD und Linken zu Arbeit/Soziales finden sich auch Resultate, die in dieser Form nicht unbedingt absehbar waren, wie die allgemein niedrige Bewertung des Politikfelds Justiz oder die vergleichsweise geringe Bedeutung des Politikfelds Kultus durch die CDU/CSU.

Wichtig ist neben den inhaltlichen Erkenntnissen über die Schwerpunkte der einzelnen Parteien und die Relevanz einzelner Politikfelder allgemein, dass eine Grundlage geschaffen wurde, auf der die Prozesse der Ämteraufteilung sinnvoll abgeschätzt werden können. Auf dieser Grundlage können auch aus anderen Quellen wie Wahlprogrammen abgeleitete Parteischwerpunkte systematisch auf Verzerrungen überprüft werden. Zudem erlauben die hier bereit gestellten Informationen, in weiteren Arbeiten systematisch Prozesse der Ämteraufteilung zu analysieren. Dies ist auch für allgemeine Koalitionstheorien relevant, die die Komponente der Ämtermotivation nicht nur rein quantitativ erfassen, sondern deren Verbindung mit der Politikmotivation berücksichtigen. ${ }^{37}$ Zusätzlich zu inhaltlichen Übereinstimmungen können Tauschpotenziale von Parteien identifiziert werden. So stehen beispielsweise SPD und Linke bei der Ämteraufteilung in gemeinsamen Regierungen in starker Konkurrenz um die in den Politikfeldern Arbeit/Soziales, Kultus und Finanzen angesiedelten Ressorts. Die Schwerpunkte von SPD und Grünen sind stärker komplementär, was sich positiv auf gemeinsame Regierungsbildungen auswirken dürfte.

37 Vgl. Eric Linhart / Franz U. Pappi, Koalitionsbildungen zwischen Ämter- und Politikmotivation. Konstruktion einer interdependenten Nutzenfunktion, in: PVS, 50. Jg. (2009), H. 1, S. 23 - 49. 